\title{
Time-Resolved Resonance Raman Spectroscopy of Excited Triplet State All-Trans-Retinal: Excitation Profiles, Intersystem Crossing Kinetics, and $\mathrm{O}_{2}$ Quenching
}

\author{
G. H. ATKINSON and J. B. PALLIX
}

Department of Chemistry, Syracuse University, Syracuse, New York 13210

The time-resolved resonance Raman $\left(\mathrm{TR}^{3}\right)$ spectra of excited triplet state all-transretinal (ATR $\left(T_{1}\right)$ ) are analyzed with respect to (1) time-resolved excitation profiles (TREP), (2) $T_{1} \rightarrow S_{0}$ intersystem crossing rates, and (3) $\mathrm{O}_{2}$ quenching kinetics. These $\mathrm{TR}^{3}$ data are used to comment on the electronic characteristics and kinetic decay of the excited triplet state of a specific retinal isomer in room temperature solutions.

\section{INTRODUCTION}

The excited state photoisomerization of retinal has been identified as a fundamental event in the visual process in general and in the functioning of the chromophore rhodopsin specifically. ${ }^{1-3}$ In spite of this important biochemical connection, there are many questions concerning the excited-state properties of retinal isomers which remain unanswered. Of particular interest here is the identification of the excited-state processes and structures which contribute to the stereoselective nature of retinal isomerization. Interest in these phenomena has already stimulated many investigations into the vibrational and electronic structure and dynamical properties of retinal..$^{4-18}$ For example, cw resonance Raman spectroscopy has been widely used to examine the vibrational structure of stable, ground-state 
retinal isomers because of its sensitivity to molecular conformations. ${ }^{4,5,7,9,12-15,17}$ To examine the excited-state structure and dynamics of a photolabile system such as retinal, however, it is essential to monitor simultaneously its molecular conformation and kinetic properties.

Recently, it was shown that these types of data can be obtained through time-resolved resonance Raman $\left(\mathrm{TR}^{3}\right)$ spectroscopy. ${ }^{19,20} \mathrm{TR}^{3}$ spectroscopy has, in fact, been used to record the vibrational spectrum of the lowest-energy, excited triplet state of all-trans-retinal $\left(\operatorname{ATR}\left(T_{1}\right)\right)^{16,18}$ It has also been recently demonstrated that the kinetic information available from $\mathrm{TR}^{3}$ spectra can be quantitatively analyzed. ${ }^{21}$ In this paper, we seek to analyze the $T^{3}$ spectra of ATR $\left(T_{1}\right)$ by examining the time-resolved excitation profile (TREP) of the major resonance Raman band from ATR $\left(T_{1}\right)$, the kinetics associated with the decay of ATR $\left(T_{1}\right)$ by $T_{1} \rightarrow S_{0}$ intersystem crossing, and the $\mathrm{O}_{2}$ quenching properties of $\operatorname{ATR}\left(T_{1}\right)$.

\section{EXPERIMENTAL}

A detailed description of the instrumentation is presented elsewhere. ${ }^{18-22}$ Briefly, $\mathrm{TR}^{3}$ spectra are recorded by synchronizing two pulsed laser systems. The initial (excitation) pulse $(354.7 \mathrm{~nm}, 1.5 \mathrm{~mJ}$ pulse, $15 \mathrm{~ns}$ pulsewidth) pumps the ground-state of ATR $\left(S_{0}\right)$ into the lowest-energy, excited singlet state, ATR $\left(S_{1}\right)$. The lowest-energy triplet state $\left(T_{1}\right)$ is subsequently populated by intersystem crossing from $S_{1}$. The second (probe) laser pulse (typically $470 \mathrm{~nm}$, $1.5 \mathrm{~mJ} /$ pulse, $8 \mathrm{~ns}$ pulsewidth) generates resonance Raman scattering from the time-dependent $T_{1}$ population. Resonance enhancements are obtained from the transient triplet-triplet absorption transition $\left(T_{1} \rightarrow T_{n}\right)$. The time delay between the arrival of the two laser beams at the sample cell can be varied between $40 \mathrm{~ns}$ and $100 \mathrm{~ms}$. The time-dependent population of the excited triplet state can be monitored by measuring the intensity of any triplet-state resonance Raman band as a function of the delay time.

The $\mathrm{TR}^{3}$ scattering is focused onto the slit of a one meter spectrograph equipped with an 1800 line per $\mathrm{mm}$ holographically-ruled grating. $\mathrm{TR}^{3}$ scattering is detected by a doubly intensified vidicon camera located in the focal plane of the spectrograph. The response 
of the vidicon is recorded digitally for storage and analysis by an on-site computer.

ATR was obtained from Eastman (purity $>97 \%$ ) and used without further purification. Samples were characterized by absorption and cw Raman spectra which duplicated spectra from the literature. Samples were stored in the dark at $5^{\circ} \mathrm{C}$ under nitrogen, but examined at room temperature. Deaerated samples were flushed with dry nitrogen for about 30 minutes prior to experiments. Concentrations of approximately $2.5 \times 10^{-4} \mathrm{M}$ were found to be optimal for the static sample conditions used.

\section{RESULTS}

The $\mathrm{TR}^{3}$ spectra of ATR $\left(T_{1}\right)$ in both methanol and benzene solvents were reported earlier. ${ }^{16,18}$ Although in principle any of these $\mathrm{TR}^{3}$ bands can serve to measure an excitation profile or as a monitor for the kinetic properties of $\operatorname{ATR}\left(T_{1}\right)$, in this work, only the strongest bands exhibited signal-to-noise ratios large enough for analysis. The $1550 \mathrm{~cm}^{-1}$ (benzene solvent) and $1555 \mathrm{~cm}^{-1}$ (methanol solvent) were examined in detail.

\section{Time-resolved excitation profile (TREP)}

The identification of $\mathrm{TR}^{3}$ scattering with the excited state of a given isomer is based on several observations including the quantitative decay properties to be analyzed here. Initially, however, we consider the TREP derived from the resonantly-enhanced nature of the Raman scattering. Excitation profiles, obtained by measuring the relative intensity of resonance Raman scattering as a function of the frequency of the exciting laser, have been used to characterize resonance Raman spectra involving stable, ground-state species (see for example Ref. 11). Comparisons of excitation profiles with absorption spectra characterize the vibronic coupling involved in resonantly-enhanced Raman scattering to the ground electronic state. The same type of information can be obtained for transient, excited-state species through a TREP. In the case of a transient scatterer, the wavelength of the probe laser is changed while the time delay between the probe 
and excitation laser pulses is held constant. To obtain data which are proportional only to the resonance Raman cross section, attention must be given to normalizing experimental parameters such as the excitation and probe laser power, illumination geometry, $\nu^{4}$ dependence of Raman scattering, and wavelength dependence of the monochromator/vidicon sensitivity. In addition, attention must be given to the transient absorption properties of the sample. These factors can be treated by ratioing the integrated area of the $\mathrm{TR}^{3}$ band to that of a normal Raman band from the solvent (see reference 21 for a more detailed analysis).

A TREP was measured for ATR $\left(T_{1}\right)$ in both methanol and benzene solvents. Figure 1 presents the TREP for the $1555 \mathrm{~cm}^{-1}$ band in the $\mathrm{TR}^{3}$ spectrum of ATR $\left(T_{1}\right)$ in methanol. The relative intensity (normalized to the methanol Raman band at $1460 \mathrm{~cm}^{-1}$ ) of this $\mathrm{TR}^{3}$ band was measured as a function of the probe laser wavelength over the range from 450 to $510 \mathrm{~nm}$. The time delay between the excitation and probe laser pulses was held constant at $40 \mathrm{~ns}$. The TREP was measured for ATR $\left(T_{1}\right)$ in benzene using the $1550 \mathrm{~cm}^{-1}$ band and probe laser wavelengths between 470 and $520 \mathrm{~nm}$. The relative intensities of the $1550 \mathrm{~cm}^{-1}$ band (normalized to the benzene Raman band at $1585 \mathrm{~cm}^{-1}$ ) increased monotonically as the probe laser wavelength approached the maximum of the transient, triplet-triplet absorption spectrum of ATR $\left(T_{1}\right)$ near $480 \mathrm{~nm}^{23}$ The TREP could not be measured at wavelengths shorter than $470 \mathrm{~nm}$ since the probe laser radiation becomes energetic enough to populate ATR $\left(T_{1}\right)$ by itself (i.e., $S_{0} \rightarrow S_{1}$ excitation followed by $S_{1} \rightarrow T_{1}$ intersystem crossing). The population of ATR $\left(T_{1}\right)$ becomes apparent by the appearance of a Raman band at $1550 \mathrm{~cm}^{-1}$ obtained with probe laser excitation $(\lambda>470 \mathrm{~nm})$ only. The increased $S_{1} \rightarrow T_{1}$ intersystem crossing yield of ATR in nonpolar solvents accounts for the presence of this phenomenon in benzene solvent and not in methanol solvent. ${ }^{24}$

The preresonance Raman excitation profile (PREP) for the $1570 \mathrm{~cm}^{-1}$ band from ATR $\left(S_{0}\right)$ in methanol was also measured using laser wavelengths between 450 and $520 \mathrm{~nm}$ (Figure 1). The relative intensities of the $1550 \mathrm{~cm}^{-1}$ band were normalized to the $1460 \mathrm{~cm}^{-1}$ Raman band of methanol. These PREP data, duplicating work reported previously, ${ }^{11}$ are in excellent agreement with the theoretical prediction based on $A$ term enhancement ${ }^{25,26}$ from a single electronic state centered at $383 \mathrm{~nm}$ (dotted curve in Figure 1 for $\bigcirc$ data points). 


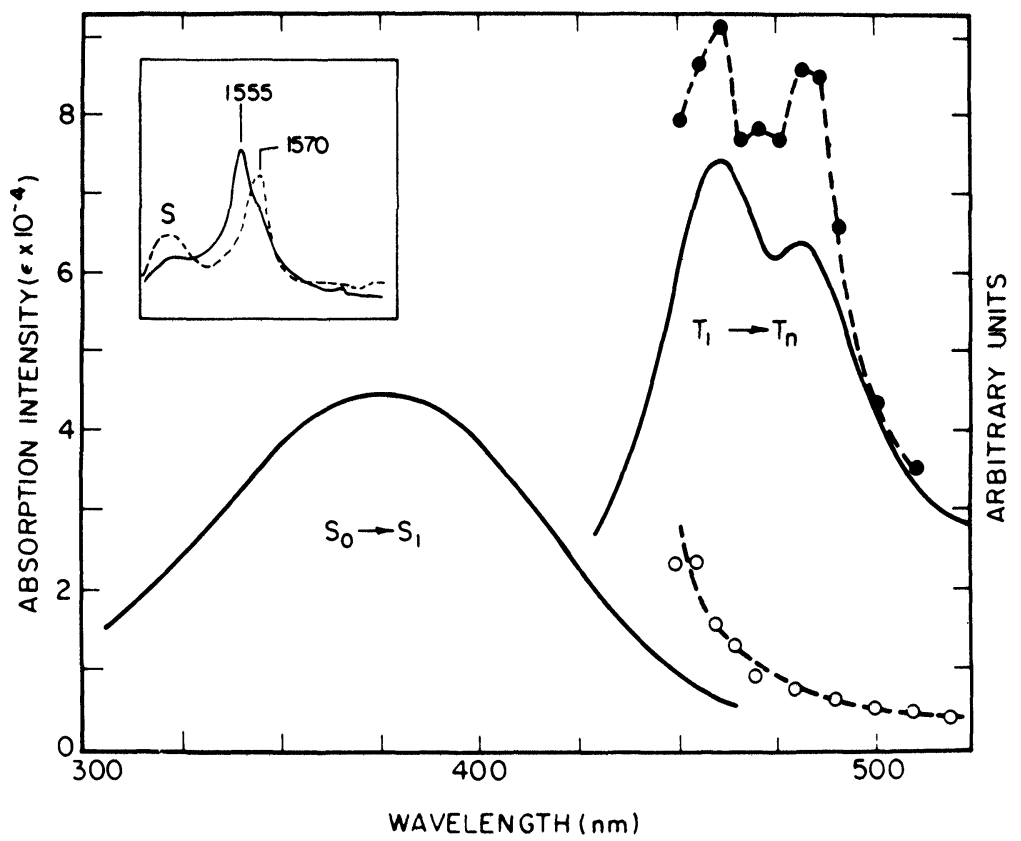

FIGURE 1 Excitation profiles for ATR $\left(T_{1}\right)$ and ATR $\left(S_{0}\right)$ bands. A preresonance excitation profile (PREP) for the $1570 \mathrm{~cm}^{-1}$ band of ATR $\left(S_{0}\right)(O$ data) and a timeresolved (40 ns delay) excitation profile (TREP) for the $1555 \mathrm{~cm}^{-1}$ of ATR $\left(T_{1}\right)($ data) are plotted with respect to the wavelength of the probe laser. The corresponding $S_{0} \rightarrow S_{1}$ and transient $T_{1} \rightarrow T_{n}$ absorption bands are also shown. All data were obtained for ATR dissolved in methanol. Insert: pulsed resonance Raman spectrum (probe laser only) using $470.0 \mathrm{~nm}$ excitation (-- - data); $\mathrm{TR}^{3}$ spectrum taken $40 \mathrm{~ns}$ after excitation of ATR with $354.7 \mathrm{~nm}$ radiation and with probe laser at $470.0 \mathrm{~nm}$ (— data). Both spectra were obtained on a $2.5 \times 10^{-4} \mathrm{M}$ sample of ATR $\left(S_{0}\right)$ in methanol. The position of the Raman band from the methanol solvent is indicated by $S$. The $1555 \mathrm{~cm}^{-1}$ resonance Raman band from ATR $\left(T_{1}\right)$ and the $1570 \mathrm{~cm}^{-1}$ Raman band from ATR $\left(S_{0}\right)$ are also labeled. ${ }^{18}$

Both the TREP and PREP are presented with the respective absorption spectra $\left(T_{1} \rightarrow T_{n}\right.$ and $\left.S_{0} \rightarrow S_{1}\right)$ from which resonance enhancements are obtained. The relative intensities of the excitation profile data were scaled to the absorption data for comparison purposes. The Raman bands used in these excitation profiles (i.e., for $\operatorname{ATR}\left(T_{1}\right)$ and ATR $\left.\left(S_{0}\right)\right)$ are presented in the inset of Figure 1. 


\section{Kinetic analysis of deaerated samples}

A general method for extracting quantitative kinetic information directly from $\mathrm{TR}^{3}$ data was recently described in the analysis of the formation and decay of excited triplet state chrysene. ${ }^{21}$ These results are used to treat the decay of ATR $\left(T_{1}\right)$ considered here. Specifically, the parameter $\Gamma$ is derived from the relative intensities of $T^{3}$ bands while the parameter $\Omega$ is defined in terms of the Raman band intensities of the solvent and measures the internal transient absorption (ITA) properties of the sample. ${ }^{21}$ Since both $\Gamma$ and $\Omega$ are proportional to the time dependent concentration of the transient, ${ }^{21}$ both can be used to measure the kinetics associated with the population of $\operatorname{ATR}\left(T_{1}\right)$.

In deaerated samples, four major decay routes for ATR $\left(T_{1}\right)$ must be considered:

$$
\begin{array}{rll}
\operatorname{ATR}\left(T_{1}\right) & \stackrel{k_{p}}{\longrightarrow} \operatorname{ATR}\left(S_{0}\right)+h \nu & \text { phosphorescence } \\
\operatorname{ATR}\left(T_{1}\right) \stackrel{k_{\text {isom }}}{\longrightarrow} \text { other retinal isomers } & \text { isomerization } \\
\operatorname{ATR}\left(T_{1}\right) \stackrel{k_{\text {isc }}}{\longrightarrow} \operatorname{ATR}\left(S_{0}\right)^{*} & \text { intersystem crossing } \\
2\left(\operatorname{ATR}\left(T_{1}\right)\right) & \stackrel{k_{a}}{\longrightarrow} 2\left(\operatorname{ATR}\left(S_{0}\right)\right) & \text { triplet-triplet annihilation }
\end{array}
$$

where ATR $\left(S_{0}\right)^{*}$ represents the vibrationally-excited, ground state of all-trans-retinal.

Phosphorescence (process 1) has been shown not to occur in retinals in solution at room temperature (weak phosphorescence has been observed at $77 \mathrm{~K}$ for retinal isomers ${ }^{27}$ ). Isomerization (process 2 ) can also be eliminated from consideration as a major decay pathway. Photoisomerization resulting from direct excitation of ATR $\left(S_{1}\right)$ in nonpolar solvents is known to have a yield of only about $0.1,{ }^{28}$ and ATR exhibits no triplet-sensitized isomerization. ${ }^{29}$

The two remaining pathways, processes (3) and (4), are both expected to contribute significantly to $\operatorname{ATR}\left(T_{1}\right)$ decay. The relative importance of triplet-triplet annihilation should be largest at early decay times since it exhibits a second-order dependence on the concentration of ATR $\left(T_{1}\right)$ while the first-order kinetics of $T_{1} \rightarrow S_{0}$ intersystem crossing should be most evident at longer decay times.

When each $\ln \Gamma$ and $\ln \Omega$ were plotted against reaction time, no single, first-order rate coefficient could be found to fit the data over the full 40 ns to $20 \mu$ s region. Rather, for times between 5 and $20 \mu$ s, 
first-order rate coefficients of $1.23 \times 10^{5} \mathrm{~s}^{-1}\left(\mathrm{TR}^{3}\right.$ data) and $1.15 \times$ $10^{5} \mathrm{~s}^{-1}$ (ITA data) were found to fit these data (Figure 2 and Table I). These values are in excellent agreement with each other (as predicted from the kinetic analysis ${ }^{21}$ ) and with values reported previously from triplet-triplet absorption experiments ${ }^{16,24}$ (Table I).

The analysis of the shorter time region ( $40 \mathrm{~ns}$ to $1 \mu \mathrm{s})$ of decay is complicated since both processes (3) and (4) must be considered. In order to evaluate the contribution of the second-order rate coefficient from triplet-triplet annihilation, the absolute volume from which $\mathrm{TR}^{3}$ and ITA data are collected must be known. Since this volume could not be measured directly, an analysis separating first and second order processes was not made. It is estimated that second-order contribu-

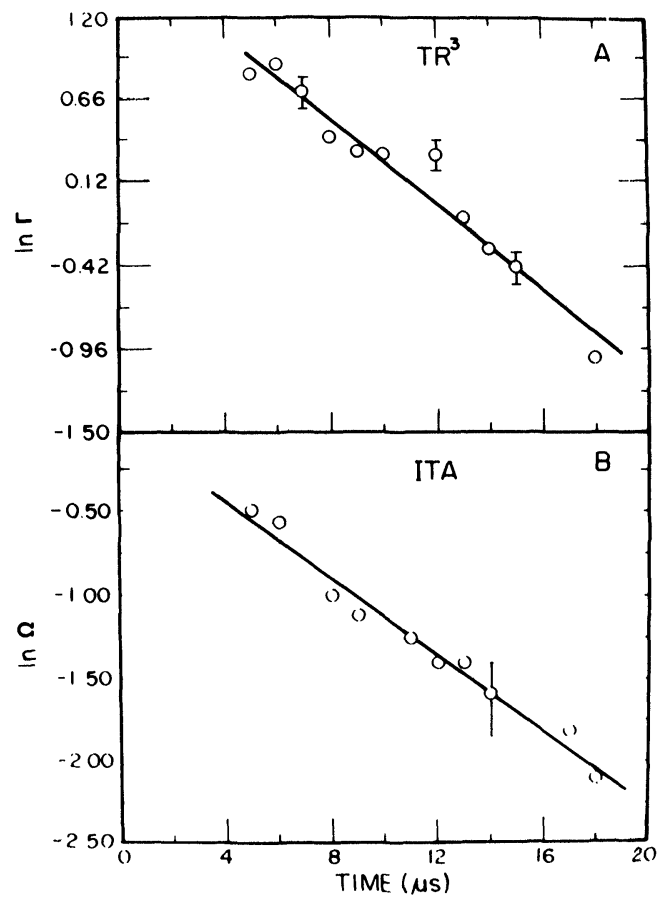

FIGURE 2 Decay kinetics of ATR $\left(T_{1}\right)$ in deaerated benzene solution. (a) $\mathrm{TR}^{3}$ data for $1550 \mathrm{~cm}^{-1}$ band of ATR $\left(T_{1}\right)$ plotted as $\ln \Gamma$ versus time; $k_{\mathrm{isc}}=1.23 \times 10^{5} \mathrm{~s}^{-1}$. (b) ITA data for $993 \mathrm{~cm}^{-1}$ band of benzene plotted as $\ln \Omega$ versus time; $k_{\text {isc }}=1.15 \times 10^{5} \mathrm{~s}^{-1}$. See Ref. 21 for definitions of $\Gamma$ and $\Omega$. 
TABLE I

Rate coefficients for the decay of all-trans-retinal

\begin{tabular}{|c|c|c|c|}
\hline Decay process & Rate coefficient & Method & Reference \\
\hline \multirow[t]{4}{*}{$\begin{array}{l}T_{1} \rightarrow S_{0} \text { intersystem } \\
\text { crossing }\left(k_{\text {isc }}\right)^{\mathrm{a}}\end{array}$} & $1.1 \times 10^{5} \mathrm{~s}^{-1}$ & $\begin{array}{r}\text { flash photolysis/ } \\
T-T \text { absorption }\end{array}$ & 24 \\
\hline & $1.15 \times 10^{5} \mathrm{~s}^{-1}$ & $\begin{array}{l}\text { pulsed radioly- } \\
\text { sis/energy } \\
\text { transfer, } T-T \\
\text { absorption }\end{array}$ & 16 \\
\hline & $1.23 \times 10^{5} \mathrm{~s}^{-1}(5-20 \mu \mathrm{s})$ & $\mathrm{TR}^{3}$ spectroscopy & This work \\
\hline & $1.15 \times 10^{5} \mathrm{~s}^{-1}(5-20 \mu \mathrm{s})$ & ITA & This work \\
\hline \multirow[t]{3}{*}{$\mathrm{O}_{2}$ quenching $\left(k_{\mathrm{q}}\right)^{\mathrm{b}}$} & $1.9 \times 10^{9} \mathrm{M}^{-1} \mathrm{~s}^{-1 \mathrm{c}}$ & $\begin{array}{r}\text { flash photolysis/ } \\
T-T \text { absorption }\end{array}$ & 31 \\
\hline & $3.5 \times 10^{9} \mathrm{M}^{-1} \mathrm{~s}^{-1}$ & $\mathrm{TR}^{3}$ spectroscopy & This work \\
\hline & $3.3 \times 10^{9} \mathrm{M}^{-1} \mathrm{~s}^{-1}$ & ITA & This work \\
\hline
\end{tabular}

${ }^{\mathrm{a}}$ Deaerated solution, $2.5 \times 10^{-4} \mathrm{M}$ in all-trans-retinal.

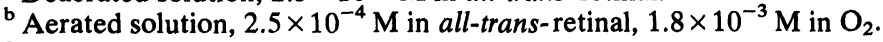

${ }^{c}$ Hexane solvent.

tions are small, however, since first-order plots for the $40 \mathrm{~ns}$ to $1 \mu \mathrm{S}$ data yield good fits (see Figure 9 Ref. 20).

\section{Kinetic analysis of oxygen quenching}

Qualitatively, the decay of $\operatorname{ATR}\left(T_{1}\right)$ has been observed to be significantly faster in the presence of molecular oxygen. ${ }^{18,20,23}$ To evaluate the quenching of ATR $\left(T_{1}\right)$ by $\mathrm{O}_{2}$ quantitatively, another process must be considered in the decay mechanism above:

$$
\operatorname{ATR}\left(T_{1}\right)+\mathrm{O}_{2} \stackrel{k_{q}}{\longrightarrow} \operatorname{ATR}\left(S_{0}\right)+\mathrm{O}_{2} \text { quenching }
$$

In the presence of air at room temperature $\left(\left[\mathrm{O}_{2}\right]=1.8 \times 10^{-3} \mathrm{M}^{30}\right)$, the decay mechanism would have contributions from processes (3), (4), and (5). The observed decay rate over the time interval 40-500 ns was found from both $\mathrm{TR}^{3}$ and ITA data to fit first-order kinetics (Figure 3). These results are to be expected for a pseudo-first order mechanism in which the concentration of $\mathrm{O}_{2}$ is large relative to that for ATR $\left(T_{1}\right)$. When the concentration of $\mathrm{O}_{2}$ dissolved in benzene is 


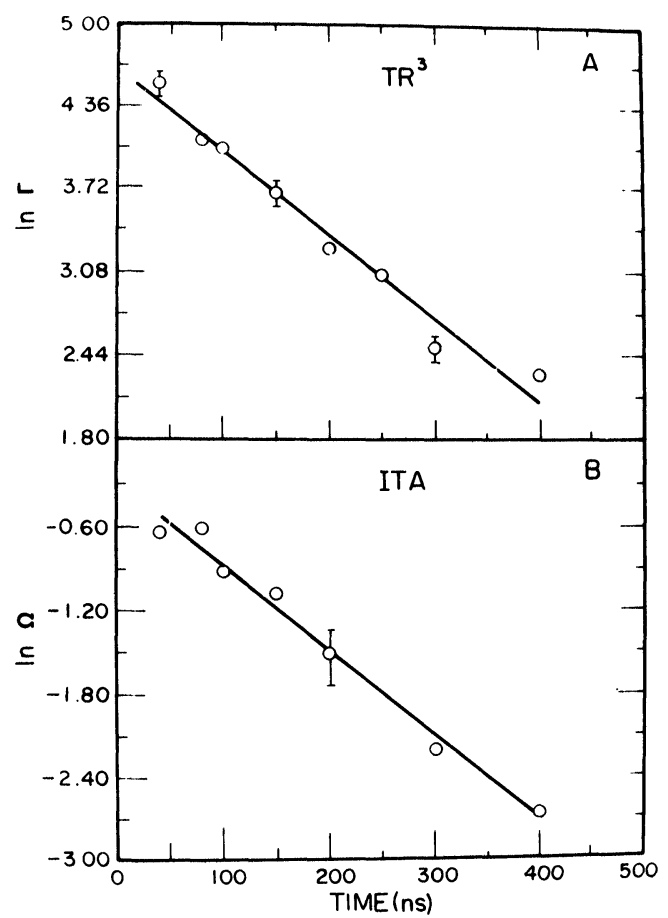

FIGURE 3 Decay kinetics of ATR $\left(T_{1}\right)$ in oxygen-saturated benzene solution. The oxygen concentration was $1.8 \times 10^{-s} \mathrm{M}$. (a) $\mathrm{TR}^{3}$ data for the $1550 \mathrm{~cm}^{-1}$ band of ATR $\left(T_{1}\right)$ plotted as $\ln \Gamma$ versus time; $k_{q}^{\prime}=k_{q}\left[\mathrm{O}_{2}\right]=6.4 \times 10^{6} \mathrm{~s}^{-1}$. (b) ITA data for the $993 \mathrm{~cm}^{-1}$ band of benzene plotted as $\ln \Omega$ versus time; $k_{q}^{\prime}=6.0 \times 10^{6} \mathrm{~s}^{-1}$. See Ref. 21 for definitions of $\Gamma$ and $\Omega$.

considered, one obtains rate coefficients for $\mathrm{O}_{2}$ quenching $\left(k_{q}\right)$ of $3.5 \times 10^{9} \mathrm{M}^{-1} \mathrm{~s}^{-1}\left(\mathrm{TR}^{3}\right)$ and $3.3 \times 10^{9} \mathrm{M}^{-1} \mathrm{~s}^{-1}$ (ITA). These values for $k_{q}$ are in reasonable agreement with a value of $1.9 \times 10^{9} \mathrm{M}^{-1} \mathrm{~s}^{-1}$ recorded in flash photolysis experiments using a different solvent (hexane versus benzene) ${ }^{31}$ (Table I).

\section{DISCUSSION}

The kinetic analysis of $\mathrm{TR}^{3}$ data for a molecule with a complex vibrational spectrum such as ATR presents several fundamental ques- 
tions which need to be addressed. Initially, an assignment of $\mathrm{TR}^{3}$ bands to a specific transient species must be made. In the case of ATR, Raman scattering was observed simultaneously from ATR $\left(S_{0}\right)$ and ATR $\left(T_{1}\right)$ (Figures 1 and 2 in Ref. 18). The distinct excitation profiles (PREP versus TREP) shown in Figure 1 provide an excellent criterion by which to assign the Raman bands in these spectra to a specific electronic state of the isomer. In the case treated here, the TREP definitively identifies the $1555 \mathrm{~cm}^{-1}$ (methanol solvent) and $1550 \mathrm{~cm}^{-1}$ (benzene solvent) bands as originating in ATR $\left(T_{1}\right)$ while the PREP confirms the ATR $\left(S_{0}\right)$ nature of the $1570 \mathrm{~cm}^{-1}$ band.

$\mathrm{TR}^{3}$ spectra, together with their associated TREP, are also sensitive to changes in excited-state bonding and the energetic ordering of excited electronic states. The sensitivity of the TREP to the solvent is evident in the different TREP observed for methanol and benzene (vide infra). These changes have been attributed both to the influence of the solvent on the relative energies of the ${ }^{1}\left(n, \pi^{*}\right)$ and ${ }^{3}\left(n, \pi^{*}\right)$ electronic states ${ }^{32}$ and to the formation of an excited-state complex between ATR $\left(T_{1}\right)$ and hydrogen-bonding solvents. ${ }^{33}$ Although a systematic use of $\mathrm{TR}^{3}$ spectra and TREP should distinguish between these two descriptions, the TREP for the bands in the $1550 / 1555 \mathrm{~cm}^{-1}$ region alone cannot. Several TREP for a variety of resonance Raman bands are needed to fully characterize any of these transient absorption bands. Transformation theory ${ }^{34}$ offers a conceptual framework by which the electronic absorption spectrum can be related to TREP so that one can be derived from the other. The use of transformation theory with a TREP could further identify and characterize the types of perturbed excited electronic states and excited-state conformers which have been used to describe ATR $\left(T_{1}\right)$. More extensive use of these types of data, however, are required before detailed conclusions can be reached.

In the presence of $\mathrm{O}_{2}$, the decay of $\operatorname{ATR}\left(T_{1}\right)$ can be interpreted in terms of a quenching scheme involving a collision complex:

$$
\begin{gathered}
\operatorname{ATR}\left(T_{1}\right)+\mathrm{O}_{2} \underset{k_{\text {dis }}}{\stackrel{k_{\text {dif }}}{\rightleftarrows}}\left\{\operatorname{ATR}\left(T_{1}\right) \cdots \mathrm{O}_{2}\right\} \stackrel{k_{\text {et }}}{\longrightarrow} \\
\operatorname{ATR}\left(S_{0}\right)+\mathrm{O}_{2}
\end{gathered}
$$

The first-order rate measured in a plot of $\ln \Gamma$ versus time is dependent on the rate coefficients for (1) diffusion to form a complex $\left(k_{\mathrm{diff}}\right),(2)$ 
dissociation of the complex $\left(k_{\mathrm{dis}}\right)$, and (3) the energy transfer process itself $\left(k_{\mathrm{et}}\right)$. Under conditions where the collision complex is in equilibrium with ATR $\left(T_{1}\right)$ and $\mathrm{O}_{2}$ (expression 6), the observed rate can be shown to equal:

$$
k_{\text {obs }}=k_{\text {dif }}\left[\mathrm{O}_{2}\right] \phi_{q}
$$

where $\phi_{q}$ is the quantum yield for energy transfer during the quenching process. The rate coefficient for diffusion of ATR $\left(T_{1}\right)$ and $\mathrm{O}_{2}$ (assuming a spherical shape for ATR of radius $7.5 \AA$ ) was calculated to be $3.1 \times 10^{10} \mathrm{M}^{-1} \mathrm{~s}^{-1}$. Expression (7) yields a value for $\phi_{q}$ of 0.11 indicating that only a small percentage of the encounters between ATR $\left(T_{1}\right)$ and $\mathrm{O}_{2}$ result in excited triplet state quenching. The low value of $\phi_{q}$ suggests that the dissociation of the collision complex dominates its decay mechanism. Presumably this inefficiency for quenching can be associated with the low viscosity of the solvents $\left(\right.$ at $25^{\circ} \mathrm{C}$, methanol; $\eta=0.55 \mathrm{cp}$ and benzene; $\eta=0.61 \mathrm{cp}$ ). It has been observed previously that $\phi_{q}$ is small for solvents with $\eta<3 \mathrm{cp}^{35}$

\section{Acknowledgement}

The authors wish to gratefully acknowledge the technical assistance of Mr. D. Gilmore. This work was supported by grants from the National Science Foundation and the National Institutes of Health.

\section{References}

1. G. Wald, Nature 219, 800 (1968).

2. B. Honig and T. G. Ebrey, Annu. Rev. Biophys. Bioeng. 3, 151 (1974).

3. T. G. Ebrey and B. Honig, Quart. Rev. Biophys. 8, 129 (1975).

4. D. Gill, M. E. Heyde and L. Rimai, J. Am. Chem. Soc. 93, 6288 (1971).

5. L. Rimai, D. Gill and J. Parsons, J. Am. Chem. Soc. 93, 53 (1971).

6. R. Bensasson, E. J. Land and T. G. Truscott, Photochem. Photobiol. 17, 53 (1973).

7. R. E. Cookingham, A. Lewis, D. Collins and M. Marcus, J. Am. Chem. Soc. 98, 2759 (1976).

8. R. M. Hochstrasser, D. L. Narva and A. C. Nelson, Chem. Phys. Lett. 43, 15 (1976).

9. R. H. Callender, A. Doukas, R. Crouch and K. Nakanishi, Biochemistry 15, 1621 (1976).

10. T. Takemura, P. K. Das, G. Hug and R. S. Becker, J. Am. Chem. Soc. 100, 9 (1978).

11. A. G. Doukas, B. Aton, R. H. Callender and B. Honig, Chem. Phys. Lett. 56, 248 (1978).

12. P. K. Das and R. S. Becker, J. Am. Chem. Soc. 101, 6348 (1979). 
13. R. Mathies, Chemical and Biochemical Applications of Lasers, Vol. 4, ed C. B. Moore (Academic Press, New York, 1979).

14. G. Eyring, B. Curry, R. Mathies, R. Fransen, I. Palings and J. Lugtenburg, Biochem. 19, 240 (1980).

15. G. Hayward, W. Carlsen, A. Siegman and L. Stryer, Science 211, 942 (1981).

16. R. Wilbrandt and N. H. Jensen, J. Am. Chem. Soc. 103, 1036 (1981).

17. C. Hsieh, M. Nagumo, M. Nicol and M. A. El-Sayed, J. Phys. Chem. 81, 2714 (1981).

18. G. H. Atkinson, J. B. Pallix, T. B. Freedman, D. A. Gilmore and R. Wibrandt, J. Am. Chem. Soc. 103, 5069 (1981).

19. G. H. Atkinson, Time-resolved Raman spectroscopy, in: Advances in Infrared and Raman Spectroscopy, Vol. 9, eds. R. J. H. Clark and R. E. Hester (Heyden and Sons, Inc. 1982) pp. 1-62 and references therein.

20. G. H. Atkinson, Time-resolved Raman spectroscopy, in: Advances in Laser Spectroscopy, Vol. 1, eds. B. A. Garetz and J. R. Lombardi (Heyden and Sons, Inc. 1982) pp. 155-175 and references therein.

21. G. H. Atkinson, D. A. Gilmore, L. R. Dosser and J. B. Pallix, J. Phys. Chem. 86, 2305 (1982).

22. G. H. Atkinson and L. R. Dosser, J. Chem. Phys. 72, 2195 (1980).

23. J. B. Pallix, M.S. Thesis, Syracuse University, 1982.

24. M. Fisher and K. Weiss, Photochem. Photobiol. 20, 423 (1974).

25. A. C. Albrecht, J. Chem. Phys. 34, 1476 (1961).

26. A. C. Albrecht and M. C. Hutley, Chem. Phys. Lett. 55, 4438 (1971).

27. R. S. Becker, K. Inusuka, J. King and D. E. Balke, J. Am. Chem. Soc. 93, 43 (1971).

28. T. Rosenfeld, A. Alchalel and M. Ottolenghi, J. Phys. Chem. 78, 336 (1974).

29. A. Kropf and R. Hubbard, Photochem. Photobiol. 12, 249 (1970).

30. Solubilities of Inorganic and Organic Compounds, Vol. 1, Part 1, eds. H. Stephen and T. Stephen (Pergamon Press, New York, 1963) p. 573.

31. T. G. Truscott, E. J. Land and A. Sykes, Photochem. Photobiol. 17, 43 (1973).

32. P. K. Das and R. S. Becker, J. Am. Chem. Soc. 101, 6348 (1979).

33. W. Dawson and E. W. Abrahamson, J. Phys. Chem. 66, 375 (1967).

34. A. C. Albrecht, Some theoretical considerations concerning Raman scattering, in: Time-Resolved Vibrational Spectroscopy, ed. G. H. Atkinson (Academic Press, New York) in press.

35. P. J. Wagner and I. Kochevar, J. Am. Chem. Soc. 90, 2232 (1968). 